\title{
$p$ th mean almost periodic solutions to neutral stochastic evolution equations with infinite delay and Poisson jumps
}

Lili Gao ${ }^{1}$ and Litan Yan²

"Correspondence:

litan-yan@hotmail.com

${ }^{2}$ Department of Mathematics,

College of Science, Donghua University, Shanghai, P.R. China

Full list of author information is

available at the end of the article

\begin{abstract}
In this paper, we investigate the $p$ th mean almost periodic solution to a neutral stochastic evolution equation with infinite delay and Poisson jumps. We give a sufficient condition for the existence and uniqueness of $p$ th mean almost periodic solution and the condition depends on the continuity of coefficients and the power of a fractional operator. We give an example to illustrate the abstract results.
\end{abstract}

Keywords: pth mean almost periodic; Poisson jumps; Poisson almost periodic; Neutral stochastic evolution equation; Infinite delay

\section{Introduction}

The theory of almost periodicity was introduced in the 1920s by Bochner and Bohr, from then on, it has been well developed in dynamical systems and differential equations. Almost periodic solutions of stochastic differential equations driven by white Gaussian noise have been studied extensively for the reason that random fluctuations come ubiquitously with all kinds of natural and unnatural systems in the real world (see [11]). More and more people are interested in studying the almost periodic solution of stochastic differential equations since the works of Da Prato et al. $[8,18]$. The concept of $p$ th mean almost periodicity has been widely studied in stochastic differential equations (see $[5,6,10]$ ) since it was firstly considered by Bezandry [4] in 2007. However, in contrast to the extensive studies on almost periodicity of solutions to stochastic differential equations driven by Gaussian noises, there has been little systematic investigation on other systems perturbed by Poisson jump. The main reason for this, in our opinion, is that Poisson jumps destroyed maybe the recurrent property completely. Some results about this question have been presented in the existing literature [11, 17, 19]. Recently, Wang and Liu [19] defined Poisson almost periodicity and investigated the existence and uniqueness of the quadratic mean almost periodic solutions to stochastic evolution equations perturbed by Lévy noise. Liu and Sun [11], Sun and Wang [17] studied almost automorphic solutions to stochastic differential equations with Lévy noise. More work can be found in $[1,2,7,12,13,15,16]$.

Motivated by the above work, we consider the existence and uniqueness of $p$ th mean almost periodic solutions to the neutral stochastic evolution equations with infinite delay

(c) The Author(s) 2019. This article is distributed under the terms of the Creative Commons Attribution 4.0 International License (http://creativecommons.org/licenses/by/4.0/), which permits unrestricted use, distribution, and reproduction in any medium, provided you give appropriate credit to the original author(s) and the source, provide a link to the Creative Commons license, and indicate if changes were made. 
and Poisson jumps have the form

$$
\left\{\begin{aligned}
d\left[x(t)-G\left(t, x(t), x_{t}\right)\right]= & {\left[A x(t)+f\left(t, x(t), x_{t}\right)\right] d t+g\left(t, x(t), x_{t}\right) d W(t) } \\
& +\int_{\mathcal{Z}} F(t, x(t), y) \tilde{N}(d t, d y), \quad t \in \mathbb{R}, \\
x_{t_{0}}=\varphi \in \mathcal{B}, &
\end{aligned}\right.
$$

where $\mathcal{B}$ is a phase space, $A$ is a closed densely defined bounded linear operator, $G$ and $f$ are $L^{p}(\Omega ; \mathbb{K})$-valued continuous functions, $g$ and $F$ are $L\left(\Omega, L^{p}(\Omega ; \mathbb{K})\right)$-valued jointly continuous functions, $W=\{W(t)\}_{t \in \mathbb{R}}$ is a $\mathcal{Q}$-valued two-sided Wiener process, $\tilde{N}$ denotes the compensated Poisson random measure of a Poisson random measure $N_{p}$ with intensity measure $v$ and $x_{t}$ is $\mathcal{B}$-valued stochastic process.

The paper is organized as follows. In Sect. 2, we recall some definitions and lemmas. In Sect. 3, we prove two key lemmas and study the existence and uniqueness of the $p$ th mean almost periodic solution to (1.1) by means of fixed point methods and the semigroup of operators. In the last section, we show an example to illustrate our main result.

\section{Preliminaries}

In this section, we briefly introduce some basic notations and facts. Let $\mathbb{K}$ be a real separable Hilbert space with inner product $(\cdot, \cdot)_{\mathbb{K}}$. We denote $L(\mathbb{K}, \mathbb{K})$ as the space of all bounded linear operators from $\mathbb{K}$ to $\mathbb{K}$. Let $(\Omega, \mathcal{F}, P)$ be a filtered complete probability space satisfying the usual conditions. Suppose $\{p(t), t \geq 0\}$ is a $\sigma$-finite stationary $\mathcal{F}_{t}$-adapted Poisson point process taking values in a measurable space $(S, \mathcal{B}(S))$, where $\mathcal{Z} \subset S=\mathbb{R}^{d}-\{0\}$. $\tilde{\mathcal{N}}(d t, d y)=\mathcal{N}_{p}(d t, d y)-v(d y)|d t|$ denotes the compensated Poisson random measure of a Poisson random measure $\mathcal{N}_{p}$ with intensity measure $v$, which is independent of $\mathcal{Q}$ Wiener process $W$. For the definition of the Poisson jumps process and its basic properties and for further details, we refer the reader to the monograph [3]. The initial value $\varphi=\{\varphi(\theta):-\infty<\theta \leq 0\}$ is an $\mathcal{F}_{0}$-measurable $\mathcal{B}$-valued random variable independent of $\mathrm{W}$ with finite second moment. The $\mathcal{B}$-valued stochastic process $x_{t}: \Omega \rightarrow \mathcal{B}, t \in \mathbb{R}$, is defined by setting $x_{t}=\{x(t+\theta)(\omega): \theta \in(-\infty, 0]\}$.

Let $A$ be a linear operator from $\mathbb{K}$ to $\mathbb{K}$ and $\{S(t), t \geq 0\}$ be an analytic semigroup with infinitesimal generator $A$. Suppose $0 \in \rho(A)$; it is well known that, for any $\alpha \in(0,1]$, the fractional power $(-A)^{\alpha}$ can be defined under some conditions which is a closed linear operator with domain $\mathcal{D}\left((-A)^{\alpha}\right)$. Moreover, $\mathcal{D}\left((-A)^{\alpha}\right)$ is dense in $\mathbb{K}$, and the expression

$$
\|h\|_{\alpha}=\left\|(-A)^{\alpha} h\right\|_{\mathbb{K}}, \quad h \in \mathcal{D}\left((-A)^{\alpha}\right)
$$

defines a norm in $\mathcal{D}\left((-A)^{\alpha}\right)$. If $\mathbb{K}_{\alpha}$ denotes the space $\mathcal{D}\left((-A)^{\alpha}\right)$ endowed with the norm $\|\cdot\|_{\alpha}$, then the following properties are well known (see Pazy [14]).

\section{Lemma 2.1}

(1) Let $0<\alpha \leq 1$. Then $\mathbb{K}_{\alpha}$ is a Banach space.

(2) For every $0<\alpha \leq 1$ there exists $M_{\alpha}>0$ such that

$$
\left\|(-A)^{\alpha} S(t)\right\| \leq M_{\alpha} t^{-\alpha} e^{-\lambda t}
$$

for all $t>0$ and $\lambda>0$. 
(3) If $0<\beta \leq \alpha$, then the injection $\mathbb{K}_{\alpha} \hookrightarrow \mathbb{K}_{\beta}$ is continuous.

(4) For $0 \leq \alpha \leq 1$, there exists a constant $C$ such that

$$
\left\|(-A)^{-\alpha}\right\| \leq C .
$$

Definition 2.1 ([9]) The phase space $\mathcal{B}((-\infty, 0], \mathbb{K})$ (denoted simply by $\mathcal{B}$ ) is the space of $\mathcal{F}_{0}$-measurable functions mapping $(-\infty, 0]$ into $\mathbb{K}$, and endowed with a semi-norm $\|\cdot\|_{\mathcal{B}}$, such that the next assumptions hold:

(A1) If $x:(-\infty, \sigma+a) \mapsto \mathbb{K}, a>0, \sigma \in \mathbb{R}$, is continuous on $[\sigma, \sigma+a)$ and $x \in \mathcal{B}$, then for every $t \in[\sigma, \sigma+a)$ the following hold:

(a1) $x_{t} \in \mathcal{B}$;

(a2) $\|x(t)\|_{\mathbb{K}} \leq N_{1}\left\|x_{t}\right\|_{\mathcal{B}}$ for some constant $N_{1}>0$ independent of $x$;

(a3) there are two measurable functions $N_{2}, N_{3}:[0, \infty) \mapsto[0, \infty), N_{2}$ independent of $x$ such that $N_{2}$ is continuous, and $N_{3}$ is locally bounded, and

$$
\left\|x_{t}\right\|_{\mathcal{B}} \leq N_{2}(t-\sigma) \sup _{\sigma \leq s \leq t}\|x(s)\|_{\mathbb{K}}+N_{3}(t-\sigma)\left\|x_{\sigma}\right\|_{\mathcal{B}} .
$$

(A2) The space $\mathcal{B}$ is complete.

(A3) For the function $x$ satisfying (A1), its corresponding history $t \rightarrow x_{t}$ is continuous function in $t \in[\sigma, \sigma+a)$.

(A4) If $\left(y_{n}\right)_{n \in \mathbb{N}}$ is a uniformly bounded sequence in $C((-\infty, 0], \mathbb{K})$ such that $y_{n}$ admits a compact support for every $n$ and $y_{n} \rightarrow y$ in the compact-open topology, then $y \in \mathcal{B}$ and $\left\|y_{n}-y\right\|_{\mathcal{B}} \rightarrow 0$, as $n \rightarrow \infty$.

In this paper, for convenience, we assume that $\mathcal{B}_{0}=\{y \in \mathcal{B}: y(0)=0\}$ and that there exists a constant $\mathcal{L}>0$ such that

$$
\|y\|_{\mathcal{B}} \leq \mathcal{L} \sup _{\theta \leq 0}\|y(\theta)\|
$$

for each $y \in \mathcal{B}$ (see Proposition 7.1.1 in [9] for many details).

Definition 2.2 Let $S_{0}(t): \mathcal{B} \rightarrow \mathcal{B}$ be a $C_{0}$-semigroup defined by

$$
\left(S_{0}(t) y\right)(\theta)= \begin{cases}y(0), & \theta \in[-t, 0], \\ y(t+\theta), & \theta \in(-\infty,-t) .\end{cases}
$$

The phase space $\mathcal{B}$ is a fading memory space if $\left\|S_{0}(t) y\right\|_{\mathcal{B}} \rightarrow 0$ for every $y \in \mathcal{B}_{0}$, as $t \rightarrow \infty$. Moreover, $\mathcal{B}$ is a uniform fading memory space whenever $\left\|S_{0}(t)\right\|_{\mathcal{B}_{0}} \rightarrow 0$, as $t \rightarrow \infty$.

For the definition of the $\mathcal{Q}$-Wiener process and its basic properties, we refer the reader to the monograph [6] for details. We now briefly recall some basic results about Poisson almost periodic and $p$ th mean almost periodic stochastic process. For the more details on these materials we refer to Bezandry and Diagana [5] and Wang and Liu [19]. Clearly, for $p \geq 2$, the space $L^{p}(\Omega, \mathbb{K})$ is a Banach space when it is equipped with the norm

$$
\|\cdot\|_{L^{p}(\Omega, \mathbb{K})}=\left(E\|\cdot\|_{\mathbb{K}}^{p}\right)^{\frac{1}{p}} .
$$


For convenience, if there is no confusion, usually the subscripts of norms $\|\cdot\|_{\mathbb{K}}$ and $\|\cdot\|_{L^{p}(\Omega, \mathbb{K})}$ will be omitted. A stochastic process $x: \mathbb{R} \rightarrow L^{p}(\Omega ; \mathbb{K})$ is said to be continuous whenever

$$
\lim _{t \rightarrow s} E\|x(t)-x(s)\|^{p}=0 .
$$

Definition 2.3 (Bochner almost periodicity) A continuous stochastic process $x: \mathbb{R} \rightarrow$ $L^{p}(\Omega ; \mathbb{K})$ is said to be $p$ th mean almost periodic if for every sequence of real numbers $\left\{\tilde{s}_{n}\right\}$, there exist a subsequence $\left\{s_{n}\right\}$ and a continuous stochastic process $\tilde{x}: \mathbb{R} \rightarrow L^{p}(\Omega ; \mathbb{K})$ such that

$$
\lim _{n \rightarrow \infty} \sup _{t \in \mathbb{R}} E\left\|x\left(t+s_{n}\right)-\tilde{x}(t)\right\|^{p}=0 .
$$

Moreover, a continuous function $g: \mathbb{R} \times L^{p}(\Omega ; \mathbb{K}) \rightarrow L^{p}(\Omega ; \mathbb{K})$ with $(t, x) \mapsto g(t, x)$ is said to be $p$ th mean almost periodic in $t$ uniformly in compact subsets of $L^{p}(\Omega ; \mathbb{K})$ if for every sequence $\left\{\tilde{s}_{n}\right\}$ of real numbers and any compact subset $\mathcal{K} \subset L^{p}(\Omega ; \mathbb{K})$, there exist a subsequence $\left\{s_{n}\right\}$ and a continuous function $\tilde{g}: \mathbb{R} \times L^{p}(\Omega ; \mathbb{K}) \rightarrow L^{p}(\Omega ; \mathbb{K})$ such that

$$
\lim _{n \rightarrow \infty} \sup _{t \in \mathbb{R}, x \in \mathcal{K}} E\left\|g\left(t+s_{n}, x\right)-\tilde{g}(t, x)\right\|^{p}=0
$$

One needs to mention that an almost periodic process is a usual $L^{p}(\Omega ; \mathbb{K})$-valued almost periodic function, so the Bochner almost periodicity is equivalent to the Bohr almost periodicity (see [5]). We denote by $\operatorname{AP}\left(\mathbb{R} ; L^{p}(\Omega ; \mathbb{K})\right)$ the collection of all $p$ th mean almost periodic stochastic processes $x: \mathbb{R} \rightarrow L^{p}(\Omega ; \mathbb{K})$, and we denote by $\mathrm{AP}\left(\mathbb{R} \times L^{p}(\Omega ; \mathbb{K}) ; L^{p}(\Omega ; \mathbb{K})\right)$ the collection of all almost periodic functions $g: \mathbb{R} \times L^{p}(\Omega ; \mathbb{K}) \rightarrow L^{p}(\Omega ; \mathbb{K})$.

Lemma 2.2 If $x \in \mathrm{AP}\left(\mathbb{R} ; L^{p}(\Omega ; \mathbb{K})\right)$, then the mapping $t \rightarrow E\|x(t)\|^{p}$ is uniformly continuous, and there exists a constant $M_{1}>0$ such that $E\|x(t)\|^{p} \leq M_{1}$ for all $t \in \mathbb{R}$.

Let $\operatorname{CUB}\left(\mathbb{R} ; L^{p}(\Omega ; \mathbb{K})\right)$ denote the collection of all continuous and uniformly bounded stochastic processes $x: \mathbb{R} \rightarrow L^{p}(\Omega ; \mathbb{K})$. It is easy to check that $\mathrm{CUB}\left(\mathbb{R} ; L^{p}(\Omega ; \mathbb{K})\right)$ is a Banach space when it is equipped with the norm

$$
\|x\|_{\infty}=\sup _{t \in \mathbb{R}}\left(E\|x(t)\|^{p}\right)^{\frac{1}{p}} .
$$

Lemma 2.3 $\operatorname{AP}\left(\mathbb{R} ; L^{p}(\Omega ; \mathbb{K})\right) \subset \mathrm{CUB}\left(\mathbb{R} ; L^{p}(\Omega ; \mathbb{K})\right)$ is a closed subspace.

Recall that a $\sigma$-finite random measure $\mathcal{N}$ on a measurable space $\left(\mathbb{R}_{+} \times S, \mathcal{B}\left(\mathbb{R}_{+} \times S\right)\right)$ is called a Poisson random measure with intensity measure $v$ if:

(1) for every $B=\left(t_{1}, t_{2}\right] \times \mathbb{Z} \in \mathcal{B}\left(\mathbb{R}_{+} \times S\right), \mathcal{N}(B)<\infty$ has a Poisson distribution with mean $E \mathcal{N}(B)=v(Z)\left(t_{2}-t_{1}\right)$;

(2) if $B_{1}, B_{2}, \ldots$ are disjoint, the random variables $\mathcal{N}\left(B_{1}\right), \mathcal{N}\left(B_{1}\right), \ldots$ are independent.

Here $\mathbb{R}_{+}=[0, \infty]$. It is well known that a Poisson random measure $\mathcal{N}$ can be characterized by a Poisson point process $p=\{p(t), t \geq 0\}$ as follows:

$$
\mathcal{N}\left(\left(t_{1}, t_{2}\right] \times \mathcal{Z}\right):=\sum_{s \in\left(t_{1}, t_{2}\right]} 1_{\mathcal{Z}}(p(s))
$$




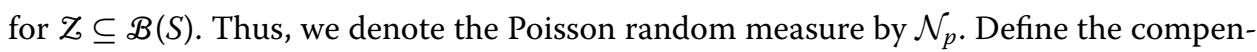
sated Poisson random measure $\tilde{\mathcal{N}}$ by

$$
\tilde{\mathcal{N}}(t, \mathbb{Z})=\mathcal{N}_{p}(t, \mathbb{Z})-t v(\mathbb{Z})
$$

Then $\tilde{\mathcal{N}}$ is a martingale valued measure. For the writing needs, we simply introduce the two-sided compensated Poisson random measure as follows: Given two independent, identically distributed Poisson point processes, define the compensated Poisson random measure

$$
\tilde{\mathcal{N}}(t, \mathcal{Z})= \begin{cases}\mathcal{N}_{p}(t, \mathcal{Z})-t v(\mathcal{Z}), & t \geq 0 \\ \mathcal{N}_{p}(-t, \mathcal{Z})+t v(\mathcal{Z}), & t<0\end{cases}
$$

Then $\tilde{\mathcal{N}}$ is a martingale valued two-sided compensated Poisson random measure.

Definition 2.4 A function $F: \mathbb{R} \times L^{p}(\Omega ; \mathbb{K}) \times \mathcal{Z} \rightarrow L^{p}(\Omega ; \mathbb{K})$ is said to be Poisson $p$ th mean almost periodic in $t \in \mathbb{R}$ uniformly in compact subsets of $L^{p}(\Omega, \mathbb{K})$ if $F$ is continuous in the following sense:

$$
\int_{\mathcal{Z}} E\|F(t, Y, x)-F(\tilde{t}, \tilde{Y}, x)\|^{p} v(d x) \rightarrow 0,
$$

as $(\tilde{t}, \tilde{Y}) \rightarrow(t, Y)$, and for every sequence $\left\{\tilde{s}_{n}\right\}$ of real numbers, there exist a subsequence $\left\{s_{n}\right\}$ and for any compact subset $\mathcal{K} \subset L^{p}(\Omega ; \mathbb{K})$, a function $\tilde{F}: \mathbb{R} \times L^{p}(\Omega ; \mathbb{K}) \times \mathcal{Z} \rightarrow L^{p}(\Omega ; \mathbb{K})$ continuous such that

$$
\lim _{n \rightarrow \infty} \sup _{t \in \mathbb{R}, Y \in \mathcal{K}} \int_{\mathcal{Z}} E\left\|F\left(t+s_{n}, Y, x\right)-\tilde{F}(t, Y, x)\right\|^{p} v(d x) \rightarrow 0 .
$$

We denoted by $\operatorname{PAP}\left(\mathbb{R} \times L^{p}(\Omega ; \mathbb{K}) \times \mathcal{Z} ; L^{p}(\Omega ; \mathbb{K})\right)$ the collection of all Poisson almost periodic functions $F: \mathbb{R} \times L^{p}(\Omega ; \mathbb{K}) \times \mathcal{Z} \rightarrow L^{p}(\Omega ; \mathbb{K})$.

The next lemma shows the basic properties of Poisson almost periodic functions.

Lemma 2.4 If $\Psi, \Psi_{1}, \Psi_{2}: \mathbb{R} \times L^{p}(\Omega ; \mathbb{K}) \times \mathcal{Z} \rightarrow L^{p}(\Omega ; \mathbb{K})$ are Poisson almost periodic functions in t uniformly in compact subsets of $L^{p}(\Omega ; \mathbb{K})$, then:

(i) $\Psi_{1}+\Psi_{2}$ is Poisson almost periodic.

(ii) $\lambda \Psi$ is Poisson almost periodic for every scalar $\lambda$.

(iii) There exists a constant $\tilde{M}=\tilde{M}(\mathcal{K})>0$ such that

$$
\sup _{t \in \mathbb{R}, Z \in \mathcal{K}} \int_{Z} E\|\Psi(t, Z, x)\|^{p} v(d x) \leq \tilde{M}
$$

for any compact subset $\mathcal{K} \subset L^{p}(\Omega ; \mathbb{K})$,

Proof It is obvious that the statements (i) and (ii) are true. For (iii), for some compact subset $\mathcal{K} \subset L^{p}(\Omega ; \mathbb{K})$, if

$$
\sup _{t \in \mathbb{R}, Z \in \mathcal{K}} \int_{\mathcal{Z}} E\|\Psi(t, Z, x)\|^{p} v(d x)=\infty
$$


then there exists a sequence $\left\{\tilde{s}_{n}\right\}$ of real numbers and a sequence $\left\{\tilde{Z}_{n}\right\} \subset \mathcal{K}$ such that

$$
\lim _{n \rightarrow \infty} \int_{\mathcal{L}} E\left\|\Psi\left(\tilde{s}_{n}, \tilde{Z}, x\right)\right\|^{p} v(d x)=\infty
$$

Since $\Psi: \mathbb{R} \times L^{p}(\Omega ; \mathbb{K}) \times \mathbb{K} \rightarrow L^{p}(\Omega ; \mathbb{K})$ is Poisson almost periodic function, there exist a subsequence $\left\{s_{n}\right\} \subset\left\{\tilde{s}_{n}\right\}$ and a function $\tilde{\Psi}: \mathbb{R} \times L^{p}(\Omega ; \mathbb{K}) \times \mathbb{K} \rightarrow L^{p}(\Omega ; \mathbb{K})$ such that

$$
\lim _{n \rightarrow \infty} \sup _{t \in \mathbb{R}, Z \in \mathcal{K}} \int_{\mathcal{Z}} E\left\|\Psi\left(t+s_{n}, Z, x\right)-\tilde{\Psi}(t, Z, x)\right\|^{p} v(d x)=0 .
$$

Notice that, for each $t \in \mathbb{R}$,

$$
\sup _{Z \in \mathcal{K}} \int_{Z} E\|\tilde{\Psi}(t, Z, x)\|^{p} v(d x)<\infty
$$

By taking $t=0$ in (2.5), we get

$$
\lim _{n \rightarrow \infty} \sup _{Z \in \mathcal{K}} \int_{\mathcal{Z}} E\left\|\Psi\left(s_{n}, Z, x\right)\right\|^{p} v(d x)<\infty
$$

which gives a contradiction. Thus the statement (iii) is true.

\section{3 th mean almost periodic mild solution}

In this section, we study the existence of $p$ th mean almost periodic mild solution to (1.1). For later use, we have to prove two key lemmas as follows.

Lemma 3.1 Let the function $f: \mathbb{R} \times L^{p}(\Omega ; \mathbb{K}) \times \mathcal{B} \rightarrow L^{p}(\Omega ; \mathbb{K})$ be pth mean almost periodic in $t \in \mathbb{R}$ uniformly in $\left(x_{1}, y_{1}\right) \in \mathbb{H} \times \mathcal{B}$, where $\mathbb{H} \subset L^{p}(\Omega ; \mathbb{K}) \times \mathcal{B}$ is compact. Iff is a Lipschitz function in the following sense:

$$
\left\|f\left(t, x_{1}, y_{1}\right)-f\left(t, x_{2}, y_{2}\right)\right\|<M_{2}\left(\left\|x_{1}-x_{2}\right\|+\left\|y_{1}-y_{2}\right\|_{\mathcal{B}}\right)
$$

for all $\left(x_{1}, y_{1}\right),\left(x_{2}, y_{2}\right) \in L^{p}(\Omega ; \mathbb{K}) \times \mathcal{B}, t \in \mathbb{R}$, and some constant $M_{2}>0$, then, for any $p$ th mean almost periodic process $\phi(t): \mathbb{R} \rightarrow L^{p}(\Omega ; \mathbb{K})$, the stochastic process $t \rightarrow f\left(t, \phi(t), \phi_{t}\right)$ is pth mean almost periodic.

Proof Let $\phi: \mathbb{R} \rightarrow L^{p}(\Omega ; \mathbb{K})$ be a $p$ th mean almost periodic process. Then, by Theorem 1.2.7 in [20], the $\mathcal{B}$-valued stochastic process $\phi_{t}=\{\phi(t+\theta):-\infty<\theta \leq 0\}$ is also $p$ th mean almost periodic. Thus, for each $\varepsilon>0$, there exists a constant $l(\varepsilon)>0$ such that every interval with the length $l(\varepsilon)$ contains a number $\tau$ satisfying

$$
E\|\phi(t+\tau)-\phi(t)\|^{p} \leq \frac{\varepsilon}{2^{2 p-1} M_{2}^{p}}, \quad \text { for all } t \in \mathbb{R}
$$

and

$$
E\left\|\phi_{t+\tau}-\phi_{t}\right\|^{p} \leq \frac{\varepsilon}{2^{2 p-1} M_{2}^{p}}, \quad \text { for all } t \in \mathbb{R} .
$$


Noting that $f$ is $p$ th mean almost periodic in $t \in \mathbb{R}$ uniformly in $\mathbb{H} \times \mathcal{B}$, we get

$$
E\left\|f\left(t+\tau, \phi(t), \phi_{t}\right)-f\left(t, \phi(t), \phi_{t}\right)\right\|^{p} \leq \frac{\varepsilon}{2^{p}}
$$

for all $t \in \mathbb{R}$ and $\varepsilon>0$. It follows that

$$
\begin{aligned}
& E\left\|f\left(t+\tau, \phi(t+\tau), \phi_{t+\tau}\right)-f\left(t, \phi(t), \phi_{t}\right)\right\|^{p} \\
&=2^{p-1} E\left(\left\|f\left(t+\tau, \phi(t+\tau), \phi_{t+\tau}\right)-f\left(t+\tau, \phi(t), \phi_{t}\right)\right\|^{p}\right. \\
&\left.\quad+\left\|f\left(t+\tau, \phi(t), \phi_{t}\right)-f\left(t, \phi(t), \phi_{t}\right)\right\|^{p}\right) \\
& \leq 2^{p-1} M_{2}^{p} E\left(\|\phi(t+\tau)-\phi(t)\|+\left\|\phi_{t+\tau}-\phi_{t}\right\|_{\mathcal{B}}\right)^{p} \\
&+2^{p-1} E\left\|f\left(t+\tau, \phi(t), \phi_{t}\right)-f\left(t, \phi(t), \phi_{t}\right)\right\|^{p} \\
& \leq 2^{2(p-1)} M_{2}^{p} E\left(\|\phi(t+\tau)-\phi(t)\|^{p}+\left\|\phi_{t+\tau}-\phi_{t}\right\|_{\mathcal{B}}^{p}\right)+2^{p-1} \frac{\varepsilon}{2^{p}} \\
& \leq \frac{\varepsilon}{2}+\frac{\varepsilon}{2}=\varepsilon
\end{aligned}
$$

for all $t \in \mathbb{R}$ and $\varepsilon>0$. Combining this with the definition of Bohr almost periodicity, we see that $f\left(t, \phi(t), \phi_{t}\right)$ is $p$ th mean almost periodic.

Lemma 3.2 Assume that $F \in \operatorname{PAP}\left(\mathbb{R} \times L^{p}(\Omega ; \mathbb{K}) \times \mathcal{Z} ; L^{p}(\Omega ; \mathbb{K})\right)$, moreover, for all $Y, \tilde{Y} \in$ $L^{p}(\Omega ; \mathbb{K}), t \in \mathbb{R}$,

$$
\int_{\mathcal{L}} E\|F(t, Y, x)-F(t, \tilde{Y}, x)\|^{p} v(d x) \leq k E\|Y-\tilde{Y}\|^{p}
$$

where constant $k>0$ is independent of $t$, then the function $D: \mathbb{R} \times \mathcal{Z} \rightarrow L^{p}(\Omega ; \mathbb{K})$ given by $D(t, x):=F(t, Y(t), x)$ is Poisson almost periodic, for any almost periodic process $Y: \mathbb{R} \rightarrow$ $L^{p}(\Omega ; \mathbb{K})$.

Proof Given a sequence $\left\{\tilde{s}_{n}\right\}$ of real numbers. For any almost periodic process $Y$, there exist a subsequence $\left\{s_{n}\right\}$ of $\left\{\tilde{s}_{n}\right\}$ and a continuous process $\tilde{Y}: \mathbb{R} \rightarrow L^{p}(\Omega ; \mathbb{K})$ such that

$$
\lim _{n \rightarrow \infty} E\left\|Y\left(t+s_{n}\right)-\tilde{Y}(t)\right\|^{p}=0
$$

uniformly in $t \in \mathbb{R}$. Since the function $F$ is Poisson almost periodic, there exist a subsequence $\left\{s_{n}\right\}$ of $\left\{\tilde{s}_{n}\right\}$ and a continuous function $\tilde{F}: \mathbb{R} \times L^{p}(\Omega ; \mathbb{K}) \times \mathcal{Z} \rightarrow L^{p}(\Omega ; \mathbb{K})$ such that

$$
\lim _{n \rightarrow \infty} \int_{\mathcal{Z}} E\left\|F\left(t+s_{n}, Y, x\right)-\tilde{F}(t, Y, x)\right\|^{p} v(d x)=\infty
$$

uniformly in $(t, Y) \in \mathbb{R} \times \mathcal{K}$, where $\mathcal{K}$ is a compact subset of $L^{p}(\Omega ; \mathbb{K})$.

Consider the function $\tilde{D}: \mathbb{R} \times \mathcal{Z} \rightarrow L^{p}(\Omega ; \mathbb{K})$ given by $\tilde{D}(t, x):=\tilde{F}(t, \tilde{Y}(t), x)$. Noting that

$$
\begin{aligned}
D\left(t+s_{n}, x\right)-\tilde{D}(t, x)= & F\left(t+s_{n}, Y\left(t+s_{n}\right), x\right) \\
& -F\left(t+s_{n}, \tilde{Y}(t), x\right)+F\left(t+s_{n}, \tilde{Y}(t), x\right)-\tilde{F}(t, \tilde{Y}(t), x),
\end{aligned}
$$


we find that

$$
\begin{array}{rl}
\int_{\mathcal{Z}} & E\left\|D\left(t+s_{n}, x\right)-\tilde{D}(t, x)\right\|^{p} v(d x) \\
\leq & 2^{p-1} \int_{\mathcal{Z}} E\left\|F\left(t+s_{n}, Y\left(t+s_{n}\right), x\right)-F\left(t+s_{n}, \tilde{Y}(t), x\right)\right\|^{p} v(d x) \\
& +2^{p-1} \int_{\mathcal{Z}} E\left\|F\left(t+s_{n}, \tilde{Y}(t), x\right)-\tilde{F}(t, \tilde{Y}(t), x)\right\|^{p} v(d x) \\
\leq & 2^{p-1} k E\left\|Y\left(t+s_{n}\right)-\tilde{Y}(t)\right\|^{p}+2^{p-1} \int_{\mathcal{Z}} E\left\|F\left(t+s_{n}, \tilde{Y}(t), x\right)-\tilde{F}(t, \tilde{Y}(t), x)\right\|^{p} v(d x)
\end{array}
$$

for every $t \in \mathbb{R}$ and $n \geq 1$. By Bochner's criterion (see [17, p. 4] for details), the closure of the range $\overline{R(Y)}$ of $Y: \mathbb{R} \rightarrow L^{p}(\Omega ; \mathbb{K})$ is a compact subset of $L^{p}(\Omega ; \mathbb{K})$, so is closure of the range $\overline{R(\tilde{Y})}$ of $\tilde{Y}$. It follows from (3.1) and (3.2) that

$$
\lim _{n \rightarrow \infty} \int_{\mathcal{L}} E\left\|D\left(t+s_{n}, x\right)-\tilde{D}(t, x)\right\|^{p} v(d x)=0
$$

uniformly in $t \in \mathbb{R}$. This completes the proof.

Definition 3.1 An $\left(\mathcal{F}_{t}\right)$-progressively process $\{x(t)\}_{t \in \mathbb{R}}$ is called a mild solution of the problem (1.1) on $\mathbb{R}$ if it satisfies the corresponding stochastic integral equation

$$
\begin{aligned}
x(t)= & S\left(t-t_{0}\right)\left[\varphi(0)-G\left(t_{0}, \varphi(0), \varphi\right)\right]+G\left(t, x(t), x_{t}\right) \\
& +\int_{t_{0}}^{t} A S(t-s) G\left(s, x(s), x_{s}\right) d s+\int_{t_{0}}^{t} S(t-s) f\left(s, x(s), x_{s}\right) d s \\
& +\int_{t_{0}}^{t} S(t-s) g\left(s, x(s), x_{s}\right) d W(s)+\int_{t_{0}}^{t} \int_{\mathcal{Z}} S(t-s) F(s, x(s), y) \tilde{N}(d s, d y)
\end{aligned}
$$

for all $t \geq t_{0}$, and $t_{0} \in \mathbb{R}$.

Now, in order to prove the main result in this paper, we introduce the following assumptions:

(H1) Let $A$ be a bounded linear operator on $\mathbb{K}$ whose associated analytic semigroup $\{S(t), t \geq 0\}$ satisfies the following:

$$
\|S(t)\| \leq M e^{-\gamma t}, \quad t \geq 0,
$$

for some $\gamma>0, M>0$.

(H2) Let the function $G: \mathbb{R} \times L^{p}(\Omega, \mathbb{K}) \times \mathcal{B} \rightarrow L^{p}\left(\Omega, \mathbb{K}_{\alpha}\right)$ be $p$ th mean almost periodic in t uniformly in compact subsets of $L^{p}\left(\Omega, \mathbb{K}_{\alpha}\right)$. Moreover, there exist a constant $\alpha \in\left(\frac{1}{p}, 1\right)$ such that, for any $\left(x_{1}, y_{1}\right),\left(x_{2}, y_{2}\right) \in L^{p}(\Omega, \mathbb{K}) \times \mathcal{B}$,

$$
\left\|(-A)^{\alpha} G\left(t, x_{1}, y_{1}\right)-(-A)^{\alpha} G\left(t, x_{2}, y_{2}\right)\right\| \leq \mathcal{L}_{G}\left(\left\|x_{1}-x_{2}\right\|+\left\|y_{1}-y_{2}\right\|_{\mathcal{B}}\right),
$$

where the constant $\mathcal{L}_{G}>0$. We further assume that $G(t, 0,0)=0$. 
(H3) Let the function $g: \mathbb{R} \times L^{p}(\Omega, \mathbb{K}) \times \mathcal{B} \rightarrow L\left(\Omega, L^{p}(\Omega, \mathbb{K})\right)$ be $p$ th mean almost periodic in t uniformly in compact subsets of $L\left(\Omega, L^{p}(\Omega, \mathbb{K})\right)$, and there exists a constant $\mathcal{L}_{g}>0$ such that

$$
\left\|g\left(t, x_{1}, y_{1}\right)-g\left(t, x_{2}, y_{2}\right)\right\| \leq \mathcal{L}_{g}\left(\left\|x_{1}-x_{2}\right\|+\left\|y-y_{2}\right\|_{\mathcal{B}}\right)
$$

for any $\left(x_{1}, y_{1}\right),\left(x_{2}, y_{2}\right) \in L^{p}(\Omega, \mathbb{K}) \times \mathcal{B}$.

(H4) Let the function $f: \mathbb{R} \times L^{p}(\Omega, \mathbb{K}) \times \mathcal{B} \rightarrow L^{p}(\Omega, \mathbb{K})$ be $p$ th mean almost periodic in $t$ uniformly in compact subsets of $L^{p}(\Omega, \mathbb{K}) \times \mathcal{B}$, and let there exist a constant $\mathcal{L}_{f}>0$ such that

$$
\left\|f\left(t, x_{1}, y_{1}\right)-f\left(t, x_{2}, y_{2}\right)\right\| \leq \mathcal{L}_{f}\left(\left\|x_{1}-x_{2}\right\|+\left\|y_{1}-y_{2}\right\|_{\mathcal{B}}\right)
$$

for any $\left(x_{1}, y_{1}\right),\left(x_{2}, y_{2}\right) \in L^{p}(\Omega, \mathbb{K}) \times \mathcal{B}$.

(H5) Let the function $F: \mathbb{R} \times L^{p}(\Omega, \mathbb{K}) \times \mathcal{Z} \rightarrow L^{p}(\Omega, \mathbb{K})$ be Poisson almost periodic in $\mathrm{t}$ uniformly in compact subsets of $L^{p}(\Omega, \mathbb{K}) \times \mathcal{Z}$, and for any $x(t), \tilde{x}(t) \in L^{p}(\Omega ; \mathbb{K})$, let there exist a constant $\mathcal{L}_{F}>0$ such that

$$
\int_{\mathcal{Z}}\|F(s, x(s-), z)-F(s, \tilde{x}(s-), z)\|^{p} v(d z) \leq \mathcal{L}_{F}\left(\|x-\tilde{x}\|^{p}\right) .
$$

The main result will be presented as below.

Theorem 3.1 Let (H1)-(H5) be satisfied. Then the problem (1.1) has a unique pth mean almost periodic mild solution whenever

$$
\begin{aligned}
\Theta:= & 5^{p-1}\left\{2 ^ { p - 1 } ( 1 + \mathcal { L } ^ { p } ) \left[C^{p}+M_{1-\alpha}^{p} \Gamma^{p-1}\left(1-\frac{p(1-\alpha)}{p-1}\right) \lambda^{-p \alpha} \mathcal{L}_{G}^{p}\right.\right. \\
& \left.\left.+\frac{M^{p}}{\gamma^{p}} \mathcal{L}_{f}^{p}+C^{\prime}(p)\left(\frac{M^{2}}{2 \gamma}\right)^{\frac{p}{2}} \mathcal{L}_{g}^{p}\right]+C(p) M^{p}\left[\left(\frac{\mathcal{L}_{F}}{2 \gamma}\right)^{\frac{p}{2}}+\frac{\mathcal{L}_{F}}{p \gamma}\right]\right\}<1,
\end{aligned}
$$

where the constants $C, M_{\alpha}$, $\lambda$ are given in Lemma 2.1 , and $\gamma, \mathcal{L}_{G}, \mathcal{L}_{g}, \mathcal{L}_{f}, \mathcal{L}_{F}$ are given in Assumptions (H1) $-(H 5)$, and $\mathcal{L}$ is given in (2.3).

Proof Clearly, the mild solution (3.3) of (1.1) is equivalent to

$$
\begin{aligned}
x(t)= & G\left(t, x(t), x_{t}\right)+\int_{-\infty}^{t} A S(t-s) G\left(s, x(s), x_{s}\right) d s \\
& +\int_{-\infty}^{t} S(t-s) f\left(s, x(s), x_{s}\right) d s+\int_{-\infty}^{t} S(t-s) g\left(s, x(s), x_{s}\right) d W(s) \\
& +\int_{-\infty}^{t} S(t-s) \int_{\mathcal{L}} F(s, x(s-), y) \tilde{N}(d s, d y) .
\end{aligned}
$$

We need to show that the above equation has a unique $p$ th mean almost periodic mild solution. Define the operator $\Lambda$ on $\operatorname{AP}\left(\mathbb{R}, L^{p}(P, \mathbb{K})\right)$ by

$$
\Lambda x(t):=G\left(t, x(t), x_{t}\right)+\int_{-\infty}^{t} A S(t-s) G\left(s, x(s), x_{s}\right) d s
$$




$$
\begin{aligned}
& +\int_{-\infty}^{t} S(t-s) f\left(s, x(s), x_{s}\right) d s+\int_{-\infty}^{t} S(t-s) g\left(s, x(s), x_{s}\right) d W(s) \\
& +\int_{-\infty}^{t} S(t-s) \int_{\mathcal{L}} F(s, x(s-), y) \tilde{N}(d s, d y) \\
= & G\left(t, x(t), x_{t}\right)+\Phi_{1} x(t)+\Phi_{2} x(t)+\Phi_{3} x(t)+\Phi_{4} x(t)
\end{aligned}
$$

for all $t \in \mathbb{R}$. We now show that $\Lambda x(t)$ is $p$ th mean almost periodic and $\Lambda$ admits a unique fixed point. This will be done in two steps.

Step I. $\Lambda x(t)$ is $p$ th mean almost periodic. By the proof of Theorem 3.1 in [10] with a minor modification, we can show that $\Phi_{1} x(t)$ and $\Phi_{2} x(t)$ are $p$ th mean almost periodic. Indeed, if $x(t)$ is $p$ th mean almost periodic, by $(H 2),(H 4)$ and Lemma 3.1, we find that $(-A)^{\alpha} G\left(s, x(s), x_{s}\right)$ and $f\left(s, x(s), x_{s}\right)$ are $p$ th mean almost periodic. Therefore, for each $\varepsilon>0$ there exists $l(\varepsilon)>0$ such that every interval with length $l(\varepsilon)$ contains a constant $\tau$ satisfying

$$
E\left\|(-A)^{\alpha} G\left(t+\tau, x(t+\tau), x_{t+\tau}\right)-(-A)^{\alpha} G\left(t, x(t), x_{t}\right)\right\|^{p}<\frac{\varepsilon \lambda^{\alpha p}}{M_{1-\alpha}^{p} \Gamma^{p}(\alpha)}
$$

and

$$
E\left\|f\left(t+\tau, x(t+\tau), x_{t+\tau}\right)-f\left(t, x(t), x_{t}\right)\right\|^{p}<\frac{\varepsilon \gamma^{p}}{M^{p}}, \quad \text { for all } t \in \mathbb{R} .
$$

By applying (2.1), (H2), the Hölder inequality and (3.7), we have

$$
\begin{aligned}
E \| & \Phi_{1} x(t+\tau)-\Phi_{1} x(t) \|^{p} \\
= & E\left\|\int_{-\infty}^{t} A S(t-s)\left[G\left(s+\tau, x(s+\tau), x_{s+\tau}\right)-G\left(s, x(s), x_{s}\right)\right] d t\right\|^{p} \\
= & E\left\|\int_{-\infty}^{t}(-A)^{1-\alpha} S(t-s)\left[(-A)^{\alpha} G\left(s+\tau, x(s+\tau), x_{s+\tau}\right)-(-A)^{\alpha} G\left(s, x(s), x_{s}\right)\right] d s\right\|^{p} \\
\leq & M_{1-\alpha}^{p}\left(\int_{-\infty}^{t} e^{-\lambda(t-s)}(t-s)^{(\alpha-1)} d s\right)^{p-1} \\
& \times\left(\int_{-\infty}^{t} e^{-\lambda(t-s)}(t-s)^{(\alpha-1)} E \|(-A)^{\alpha} G\left(s+\tau, x(s+\tau), x_{s+\tau}\right)\right. \\
& \left.-(-A)^{\alpha} G\left(s, x(s), x_{s}\right) \|^{p} d s\right) \\
\leq & M_{1-\alpha}^{p}\left(\int_{-\infty}^{t} e^{-\lambda(t-s)}(t-s)^{(\alpha-1)} d s\right)^{p} \times \frac{\varepsilon \lambda^{\alpha p}}{M_{1-\alpha}^{p} \Gamma^{p}(\alpha)} \\
\leq & \frac{\varepsilon \lambda^{\alpha p}}{\Gamma^{p}(\alpha)}\left(\int_{-\infty}^{t} e^{-\lambda(t-s)}(t-s)^{(\alpha-1)} d s\right)^{p} \leq \varepsilon, \quad \text { for all } t \in \mathbb{R} .
\end{aligned}
$$

For $\Phi_{2}$, by $(H 1),(H 4)$, the Hölder inequality and (3.8), we have

$$
\begin{aligned}
E \| & \Phi_{2} x(t+\tau)-\Phi_{2} x(t) \|^{p} \\
& =E\left\|\int_{-\infty}^{t} S(t-s)\left[f\left(s+\tau, x(s+\tau), x_{s+\tau}\right)-f\left(s, x(s), x_{s}\right)\right] d t\right\|^{p} \\
& \leq M^{p}\left(\int_{-\infty}^{t} e^{-\gamma(t-s)} d s\right)^{p-1}
\end{aligned}
$$




$$
\begin{aligned}
& \times\left(\int_{-\infty}^{t} e^{-\gamma(t-s)} E\left\|f\left(s+\tau, x(s+\tau), x_{s+\tau}\right)-f\left(s, x(s), x_{s}\right)\right\|^{p} d s\right) \\
\leq & M^{p}\left(\int_{-\infty}^{t} e^{-\gamma(t-s)} d s\right)^{p} \times \frac{\varepsilon \gamma^{p}}{M^{p}} \\
\leq & \varepsilon \gamma^{p}\left(\int_{-\infty}^{t} e^{-\gamma(t-s)} d s\right)^{p} \leq \varepsilon, \quad \text { for all } t \in \mathbb{R} .
\end{aligned}
$$

Similarly, by the proof of Theorem 5.1 in [5, pp. 131-132] with minor modifications, we find that $\Phi_{3} x(t)$ is $p$ th mean almost periodic.

Now, we will prove that $\Phi_{4} x(t)$ is almost periodic. Denote $\mathcal{D}(t, y):=F(t, x(t-), y)$ for $F \in$ $\operatorname{PAP}\left(\mathbb{R} \times L^{p}(\Omega, \mathbb{K}) \times \mathcal{Z} ; L^{p}(\Omega, \mathbb{K})\right)$ and let $\tilde{s}_{n}$ be an arbitrary sequence of real numbers. Then $\mathcal{D}(t, y)$ is Poisson almost periodic and there exist a subsequence $s_{n}$ of $\tilde{s}_{n}$ and continuous functions $\tilde{\mathcal{D}}$ such that

$$
\limsup _{n \rightarrow \infty, y \in \mathbb{R}} \int_{\mathcal{Z}} E\left\|\mathcal{D}\left(t+s_{n}, y\right)-\tilde{\mathcal{D}}(t, y)\right\|^{p} v(d y)=0
$$

Let $\tilde{\mathcal{N}}_{1}(\sigma, \cdot):=\tilde{\mathcal{N}}\left(\sigma+s_{n}, \cdot\right)-\tilde{\mathcal{N}}\left(s_{n}, \cdot\right)$ for each $\sigma \in \mathbb{R}, \tilde{\mathcal{N}}_{1}$ and $\tilde{\mathcal{N}}$ has the same law. Let $\sigma=s-s_{n}$, by Kunita's first inequality [3] and (H1), we derive

$$
\begin{aligned}
& E\left\|\int_{-\infty}^{t+s_{n}} \int_{\mathcal{Z}} S\left(t+s_{n}-s\right) \mathcal{D}(s, y) \tilde{\mathcal{N}}(d s, d y)-\int_{-\infty}^{t} \int_{\mathcal{Z}} S(t-s) \tilde{\mathcal{D}}(s, y) \tilde{\mathcal{N}}(d s, d y)\right\|^{p} \\
&=E\left\|\int_{-\infty}^{t} \int_{\mathcal{Z}} S(t-s)\left[\mathcal{D}\left(\sigma+s_{n}, y\right)-\tilde{\mathcal{D}}(\sigma, y)\right] \tilde{\mathcal{N}}(d s, d y)\right\|^{p} \\
& \leq C(p)\left\{E\left(\int_{-\infty}^{t} \int_{\mathcal{Z}}\left\|S(t-s)\left[\mathcal{D}\left(\sigma+s_{n}, y\right)-\tilde{\mathcal{D}}(\sigma, y)\right]\right\|^{2} v(d y) d s\right)^{\frac{p}{2}}\right. \\
&\left.+E\left(\int_{-\infty}^{t} \int_{\mathcal{Z}}\left\|S(t-s)\left[\mathcal{D}\left(\sigma+s_{n}, y\right)-\tilde{\mathcal{D}}(\sigma, y)\right]\right\|^{p} v(d y) d s\right)\right\} \\
& \leq C(p)\left\{\left(\int_{-\infty}^{t} \int_{\mathcal{Z}}\|S(t-s)\|^{2} E\left\|\mathcal{D}\left(\sigma+s_{n}, y\right)-\tilde{\mathcal{D}}(\sigma, y)\right\|^{2} v(d y) d s\right)^{\frac{p}{2}}\right. \\
&\left.\quad+\int_{-\infty}^{t} \int_{\mathcal{L}}\|S(t-s)\|^{p} E\left\|\mathcal{D}\left(\sigma+s_{n}, y\right)-\tilde{\mathcal{D}}(\sigma, y)\right\|^{p} v(d y) d s\right\} \\
& \leq C(p)\left\{\left(\int_{-\infty}^{t} M^{2} e^{-2 \gamma(t-\sigma)} \int_{\mathcal{L}} E\left\|\mathcal{D}\left(\sigma+s_{n}, y\right)-\tilde{\mathcal{D}}(\sigma, y)\right\|^{2} v(d y) d s\right)^{\frac{p}{2}}\right. \\
&\left.+\int_{-\infty}^{t} M^{p} e^{-p \gamma(t-\sigma)} \int_{\mathcal{Z}} E\left\|\mathcal{D}\left(\sigma+s_{n}, y\right)-\tilde{\mathcal{D}}(\sigma, y)\right\|^{p} v(d y) d s\right\}
\end{aligned}
$$

where $C(p)>0$ is a constant. Combining this with (3.9), we find that $\Phi_{4} x(t)$ is $p$ th mean almost periodic in $t$. Thus, we have showed that the operator $\Lambda x(t)$ is $p$ th mean almost periodic.

Step II. $\Lambda$ has a unique fixed point. Assume that both $x(t)$ and $y(t)$ are almost periodic solutions of (1.1), and $x(t) \neq y(t)$. We derive from (3.6) that

$$
E\|\Lambda x(t)-\Lambda y(t)\|^{p} \leq 5^{p-1}\left\{E\left\|G\left(t, x(t), x_{t}\right)-G\left(t, y(t), y_{t}\right)\right\|^{p}\right.
$$




$$
\begin{aligned}
& +E\left\|\int_{-\infty}^{t} A S(t-s)\left[G\left(s, x(s), x_{s}\right)-G\left(s, y(s), y_{s}\right)\right] d s\right\|^{p} \\
& +E\left\|\int_{-\infty}^{t} S(t-s)\left[f\left(s, x(s), x_{s}\right)-f\left(s, y(s), y_{s}\right)\right] d s\right\|^{p} \\
& +E\left\|\int_{-\infty}^{t} S(t-s)\left[g\left(s, x(s), x_{s}\right)-g\left(s, y(s), y_{s}\right)\right] d W(s)\right\|^{p} \\
& \left.+E\left\|\int_{-\infty}^{t} \int_{\mathcal{Z}} S(t-s)[F(s, x(s), z)-F(s, y(s), z)] \tilde{\mathcal{N}}(d s, d z)\right\|^{p}\right\} \\
& \equiv 5^{p-1} \sum_{i=1}^{5} \psi_{i}
\end{aligned}
$$

for all $t \in \mathbb{R}$. By (H1), (H2) and (2.2), we have

$$
\begin{aligned}
\psi_{1} & =E\left\|G\left(t, x(t), x_{t}\right)-G\left(t, y(t), y_{t}\right)\right\|^{p} \\
& \leq E\left\|(-A)^{-\alpha}\right\|^{p}\left\|(-A)^{\alpha} G\left(t, x(t), x_{t}\right)-(-A)^{\alpha} G\left(t, y(t), y_{t}\right)\right\|^{p} \\
& \leq C^{p} \mathcal{L}_{G}^{p} \sup _{t \in \mathbb{R}} E\left(\|x(t)-y(t)\|+\left\|x_{t}-y_{t}\right\|_{\mathcal{B}}\right)^{p} \\
& \leq C^{p} 2^{p-1}\left(1+\mathcal{L}^{p}\right) \mathcal{L}_{G}^{p} \sup _{t \in \mathbb{R}} E\left(\|x(t)-y(t)\|^{p}\right) \\
& \leq C^{p} 2^{p-1}\left(1+\mathcal{L}^{p}\right) \mathcal{L}_{G}^{p}\|x-y\|_{\infty}^{p} .
\end{aligned}
$$

Applying (2.2), we get

$$
\begin{aligned}
\psi_{2} & =E\left\|\int_{-\infty}^{t} A S(t-s)\left[G\left(s, x(s), x_{s}\right)-G\left(s, y(s), y_{s}\right)\right] d s\right\|^{p} \\
& =E\left\|\int_{-\infty}^{t}(-A)^{1-\alpha} S(t-s)(-A)^{\alpha}\left[G\left(s, x(s), x_{s}\right)-G\left(s, y(s), y_{s}\right)\right] d s\right\|^{p} \\
& \leq \int_{-\infty}^{t} M_{1-\alpha}(t-s)^{-(1-\alpha)} e^{-\lambda(t-s)} E\left\|(-A)^{\alpha}\left[G\left(s, x(s), x_{s}\right)-G\left(s, y(s), y_{s}\right)\right] d s\right\|^{p},
\end{aligned}
$$

by $(H 2)$ and the Hölder inequality, we have

$$
\begin{aligned}
\psi_{2} \leq & M_{1-\alpha}^{p}\left(\int_{-\infty}^{t} e^{-\lambda(t-s)}(t-s)^{\frac{p(\alpha-1)}{p-1}} d s\right)^{p-1} \\
& \times \int_{-\infty}^{t} e^{-\lambda(t-s)} E\left\|(-A)^{\alpha} G\left(s, x(s), x_{s}\right)-G\left(s, y(s), y_{s}\right)\right\|^{p} d s \\
\leq & M_{1-\alpha}^{p}\left(\Gamma\left(1-\frac{p(1-\alpha)}{p-1}\right) \lambda^{\frac{p(1-\alpha)}{p-1}-1}\right)^{p-1} \\
& \times\left(\frac{1}{\lambda}\right) \mathcal{L}_{G}^{p} \sup _{t \in \mathbb{R}} E\left(\|x(t)-y(t)\|+\left\|x_{t}-y_{t}\right\|_{\mathcal{B}}\right)^{p} \\
\leq & M_{1-\alpha}^{p}\left(\Gamma\left(1-\frac{p(1-\alpha)}{p-1}\right) \lambda^{\frac{p(1-\alpha)}{p-1}-1}\right)^{p-1} \\
& \times\left(\frac{1}{\lambda}\right) \mathcal{L}_{G}^{p} 2^{p-1} \sup _{t \in \mathbb{R}} E\left(\|x(t)-y(t)\|+\left\|x_{t}-y_{t}\right\|_{\mathcal{B}}^{p}\right)
\end{aligned}
$$




$$
\begin{aligned}
\leq & M_{1-\alpha}^{p}\left(\Gamma\left(1-\frac{p(1-\alpha)}{p-1}\right) \lambda^{\frac{p(1-\alpha)}{p-1}-1}\right)^{p-1} \\
& \times\left(\frac{1}{\lambda}\right) \mathcal{L}_{G}^{p} 2^{p-1}\left(1+\mathcal{L}^{p}\right) \sup _{t \in \mathbb{R}} E(\|x(t)-y(t)\|) \\
\leq & M_{1-\alpha}^{p}\left(\Gamma\left(1-\frac{p(1-\alpha)}{p-1}\right) \lambda^{\frac{p(1-\alpha)}{p-1}-1}\right)^{p-1} \times\left(\frac{1}{\lambda}\right) \mathcal{L}_{G}^{p} 2^{p-1}\left(1+\mathcal{L}^{p}\right)\|x-y\|_{\infty}^{p} \\
= & M_{1-\alpha}^{p} \Gamma^{p-1}\left(1-\frac{p(1-\alpha)}{p-1}\right) \lambda^{-p \alpha} \mathcal{L}_{G}^{p} 2^{p-1}\left(1+\mathcal{L}^{p}\right)\|x-y\|_{\infty}^{p}
\end{aligned}
$$

for all $t \in \mathbb{R}$. By $(H 1),(H 4)$ and the Hölder inequality, we also have

$$
\begin{aligned}
\psi_{3} & \leq E\left(\int_{-\infty}^{t}\|S(t-s)\|\left\|f\left(s, x(s), x_{s}\right)-f\left(s, y(s), y_{s}\right)\right\| d s\right)^{p} \\
& \leq\left(\int_{-\infty}^{t} M e^{-\gamma(t-s) d s}\right)^{p-1} \times \int_{-\infty}^{t} M e^{-\gamma(t-s)} E\left\|f\left(s, x(s), x_{s}\right)-f\left(s, y(s), y_{s}\right)\right\|^{p} d s \\
& \leq\left(\int_{-\infty}^{t} M e^{-\gamma(t-s)} d s\right)^{p} \sup _{t \in \mathbb{R}} E\left\|f\left(t, x(t), x_{t}\right)-f\left(t, y(t), y_{t}\right)\right\|^{p},
\end{aligned}
$$

moreover, we can easily find from $(H 4)$ that

$$
\begin{aligned}
\psi_{3} & \leq \frac{M^{p}}{\gamma^{p}} \mathcal{L}_{f}^{p} \sup _{t \in \mathbb{R}} E\left(\|x(t)-y(t)\|+\left\|x_{t}-y_{t}\right\|_{\mathcal{B}}\right)^{p} \\
& \leq \frac{M^{p}}{\gamma^{p}} \mathcal{L}_{f}^{p} 2^{p-1} \sup _{t \in \mathbb{R}} E\left(\|x(t)-y(t)\|^{p}+\left\|x_{t}-y_{t}\right\|_{\mathcal{B}}^{p}\right) \\
& \leq \frac{M^{p}}{\gamma^{p}} \mathcal{L}_{f}^{p} 2^{p-1}\left(1+\mathcal{L}^{p}\right)\|x-y\|_{\infty}^{p}
\end{aligned}
$$

for all $t \in \mathbb{R}$. By $(H 1)$ and the B-D-G inequalities, there exists a constant $C^{\prime}(p)>0$ such that

$$
\begin{aligned}
\psi_{4} & \leq C^{\prime}(p) E\left\|\int_{-\infty}^{t}\right\| S(t-s)\left[g\left(s, x(s), x_{s}\right)-g\left(s, y(s), y_{s}\right)\right]\left\|_{L^{p}(\Omega, \mathbb{K})}^{2} d s\right\|^{\frac{p}{2}} \\
& \leq C^{\prime}(p) E\left\|\int_{-\infty}^{t} M^{2} e^{-2 \gamma(t-s)}\right\| g\left(s, x(s), x_{s}\right)-g\left(s, y(s), y_{s}\right)\left\|_{L^{p}(\Omega, \mathbb{K})}^{2} d s\right\|^{\frac{p}{2}},
\end{aligned}
$$

moreover, by $(H 3)$ and an elementary inequality, we have

$$
\begin{aligned}
\psi_{4} & \leq C^{\prime}(p)\left(\int_{-\infty}^{t} M^{2} e^{-2 \gamma(t-s)} d s\right)^{\frac{p}{2}} \mathcal{L}_{g}^{p} \sup _{t \in \mathbb{R}} E\left(\|x(t)-y(t)\|+\left\|x_{t}-y_{t}\right\|_{\mathcal{B}}\right)^{p} \\
& \leq C^{\prime}(p)\left(\frac{M^{2}}{2 \gamma}\right)^{\frac{p}{2}} \mathcal{L}_{g}^{p} 2^{p-1} \sup _{t \in \mathbb{R}} E\left(\|x(t)-y(t)\|^{p}+\left\|x_{t}-y_{t}\right\|_{\mathcal{B}}^{p}\right) \\
& \leq C^{\prime}(p)\left(\frac{M^{2}}{2 \gamma}\right)^{\frac{p}{2}} \mathcal{L}_{g}^{p} 2^{p-1}\left(1+\mathcal{L}^{p}\right) \sup _{t \in \mathbb{R}} E(\|x(t)-y(t)\|) \\
& \leq C^{\prime}(p)\left(\frac{M^{2}}{2 \gamma}\right)^{\frac{p}{2}} \mathcal{L}_{g}^{p} 2^{p-1}\left(1+\mathcal{L}^{p}\right)\|x-y\|_{\infty}^{p}
\end{aligned}
$$


for all $t \in \mathbb{R}$. For $\psi_{5}$, by Kunita's inequality (see [3]), we find that there exists a constant $C(p)>0$ such that

$$
\begin{aligned}
\psi_{5} \leq & C(p)\left\{E\left(\int_{-\infty}^{t} \int_{\mathcal{Z}}\|S(t-s)[F(s, x(s), z)-F(s, y(s), z)]\|^{2} v(d z) d s\right)^{\frac{p}{2}}\right. \\
& \left.+E\left(\int_{-\infty}^{t} \int_{\mathcal{Z}}\|S(t-s)[F(s, x(s), z)-F(s, y(s), z)]\|^{p} v(d z) d s\right)\right\},
\end{aligned}
$$

we can derive from $(H 1)$ and $(H 5)$ that

$$
\begin{aligned}
\psi_{5} \leq & C(p)\left\{\left(\int_{-\infty}^{t} M^{2} e^{-2 \gamma(t-s)} \int_{\mathcal{Z}} E\|F(s, x(s), z)-F(s, y(s), z)\|^{2} v(d z) d s\right)^{\frac{p}{2}}\right. \\
& \left.+\int_{-\infty}^{t} M^{p} e^{-p \gamma(t-s)} \int_{\mathcal{Z}} E\|F(s, x(s), z)-F(s, y(s), z)\|^{p} v(d z) d s\right\} \\
\leq & C(p)\left\{\left(\frac{M^{2}}{2 \gamma} \mathcal{L}_{F} E\|x(t)-y(t)\|^{2}\right)^{\frac{p}{2}}+\frac{M^{p} \mathcal{L}_{F}}{p \gamma} E\|x(t)-y(t)\|^{p}\right\} \\
\leq & C(p)\left[\left(\frac{M^{2} \mathcal{L}_{F}}{2 \gamma}\right)^{\frac{p}{2}}+\frac{M^{p} \mathcal{L}_{F}}{p \gamma}\right] \sup _{t \in \mathbb{R}} E\|x(t)-y(t)\|^{p} \\
\leq & C(p) M^{p}\left[\left(\frac{\mathcal{L}_{F}}{2 \gamma}\right)^{\frac{p}{2}}+\frac{\mathcal{L}_{F}}{p \gamma}\right]\|x-y\|_{\infty}^{p}
\end{aligned}
$$

for all $t \in \mathbb{R}$. Thus, we have showed the desired estimate

$$
E\|\Lambda x(t)-\Lambda y(t)\|^{p} \leq \Theta\|x-y\|_{\infty}^{p}
$$

for all $t \in \mathbb{R}$, which implies that

$$
\|\Lambda x(t)-\Lambda y(t)\|_{\infty} \leq \sqrt[p]{\Theta}\|x-y\|_{\infty}
$$

for all $t \in \mathbb{R}$, i.e., $\Lambda$ is a contraction when (3.5) holds. It follows from the contraction principle that there exists a unique fixed point $x(t)$ for $\Lambda$ in $\operatorname{AP}\left(\mathbb{R} ; L^{p}(\Omega, \mathbb{K})\right)$ such that $\Lambda x=x$, which implies that (1.1) admits a unique almost periodic mild solution.

\section{Examples}

In this section, we give an example to illustrate the result obtained. First, we show a fading memory phase space $\mathcal{B}_{h}$. For any $a>0$, define

$$
\mathcal{B}=\{\psi:[-a, 0] \rightarrow \mathbb{K} \text { is bounded and measurable }\}
$$

with the norm

$$
\|\psi\|_{[-a, 0]}=\sup _{s \in[-a, 0]} E\|\psi(s)\|, \quad \psi \in \mathcal{B}
$$

and

$$
\mathcal{B}_{h}=\left\{\psi:(-\infty, 0] \rightarrow \mathbb{K} \mid \int_{-\infty}^{0} h(s) \sup _{s \leq \theta \leq 0} E(\|\psi\|) d s<\infty\right\},
$$


where $h:(-\infty, 0] \rightarrow(0, \infty)$ is a given continuous function with $l=\int_{-\infty}^{0} h(t) d t<\infty$. If $\mathcal{B}_{h}$ is endowed with the norm

$$
\|\psi\|_{\mathcal{B}_{h}}=\int_{-\infty}^{0} h(s)\|\psi\|_{[s, 0]} d s,
$$

then $\left(\mathcal{B}_{h},\|\cdot\|_{\mathcal{B}_{h}}\right)$ is a Banach space and satisfies all conditions in Definition 2.1.

Consider the neutral stochastic differential equations with infinite delays and jumps

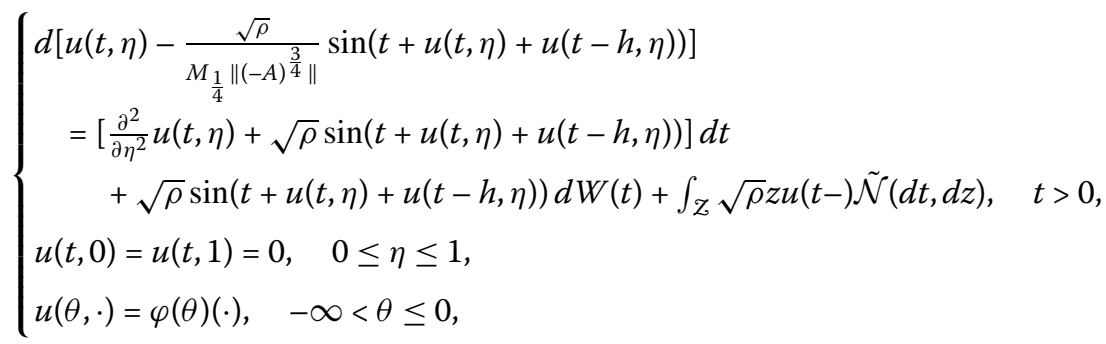

where $W(t)$ is a standard cylindrical Wiener process and $\mathcal{Z}=\{z \in \mathbb{R}: 0<|z| \leq b, b>0\}$. Let $\mathbb{K}=L^{2}[0,1]$ and the operator $A=\Delta$ be the classical Laplacian defined by

$$
(A u)(\eta)=\frac{\partial^{2} u}{\partial \eta^{2}}
$$

with domain $D(A)=\left\{u \in \mathbb{K}: u, u^{\prime}\right.$ are absolutely continous, $\left.u^{\prime \prime} \in \mathbb{K}, u(0)=u(1)\right\}$, then $A$ has eigenvalues

$$
\left\{-\pi^{2},-4 \pi^{2}, \ldots,-n^{2} \pi^{2}, \ldots\right\}
$$

and it generates an analytic semigroup $\{S(t)\}_{t \geq 0}$ on $\mathbb{K}$ satisfying $\|S(t)\| \leq e^{-\pi^{2} t}$. By taking the phase space $\mathcal{B}$ as (4.1) with $h(t)=e^{t},-\infty<t<0$ and letting

$$
\begin{aligned}
& G\left(t, x, x_{t}\right)(\eta)=\frac{\sqrt{\rho}}{M_{\frac{1}{4}}\left\|(-A)^{\frac{3}{4}}\right\|} \sin (t+u(t, \eta)+u(t-h, \eta)) \\
& f\left(t, x, x_{t}\right)(\eta)=\sqrt{\rho} \sin (t+u(t, \eta)+u(t-h, \eta)) \\
& g\left(t, x, x_{t}\right)(\eta)=\sqrt{\rho} \sin (t+u(t, \eta)+u(t-h, \eta)) \\
& F(t, x, z)(\eta)=\sqrt{\rho} z u(t, \eta)
\end{aligned}
$$

where $\rho>0, M_{\frac{1}{4}}>0$ are the two corresponding constants in Lemma 2.1, we then rewrite the system (4.2) into the abstract form (1.1). Moreover, it is not difficult to check that $G$, $f, g, F$ satisfy the conditions $(H 2)-(H 5)$ with $p=2$. Taking $\alpha=\frac{3}{4}$ and $\lambda=\pi^{1 / 3}$, we get

$$
l=\int_{-\infty}^{0} e^{t} d t=1, \quad \gamma=\pi^{2}, \quad M=1, \quad \mathcal{L}_{G}=\frac{\sqrt{\rho}}{M_{\frac{1}{4}}}
$$

and

$$
\mathcal{L}_{f}=\mathcal{L}_{g}=\sqrt{\rho}, \quad \mathcal{L}_{F}=\sqrt{\rho} \int_{\mathcal{Z}} z^{2} v(d z) .
$$


For $(-A)^{-\frac{3}{4}}($ see $[14$, p. 70]), we have

$$
\left\|(-A)^{-\frac{3}{4}}\right\| \leq \frac{1}{\Gamma\left(\frac{3}{4}\right)} \int_{0}^{\infty} t^{-\frac{1}{4}}\|S(t)\| d t \leq \pi^{-\frac{3}{2}}=C .
$$

Theorem 3.1 implies that the system (4.2) has a unique mild square mean almost periodic solution, provided

$$
4\left(\frac{1}{\pi^{3}}+\rho+\frac{\rho}{\pi^{4}}+\frac{\rho}{2 \pi^{2}}\right)+\frac{1}{\pi^{2}} \rho \int_{\mathcal{Z}} z^{2} v(d z)<\frac{1}{5} .
$$

\section{Funding}

The Project-sponsored by the National Natural Science Foundation of China (11571071), the Fundamental Research Funds for the Central Universities (No. 18D310405).

\section{Competing interests}

The authors declare that they have no competing interests.

\section{Authors' contributions}

LG and LY carried out the mathematical studies, participated in the sequence alignment, drafted the manuscript and participated in the design of the study and performed proof of results. All authors read and approved the final manuscript.

\section{Author details}

${ }^{1}$ College of Information Science and Technology, Donghua University, Shanghai, P.R. China. ${ }^{2}$ Department of Mathematics, College of Science, Donghua University, Shanghai, P.R. China.

\section{Publisher's Note}

Springer Nature remains neutral with regard to jurisdictional claims in published maps and institutional affiliations.

\section{Received: 17 August 2018 Accepted: 2 July 2019 Published online: 19 July 2019}

\section{References}

1. AhmedEmail, H.M., El-Borai, M.M., Elsaid Ramadan, M.: Boundary controllability of nonlocal Hilfer fractional stochastic differential systems with fractional Brownian motion and Poisson jumps. Adv. Differ. Equ. 2019, 82 (2019)

2. Annamalai, A., Kandasamy, B., Baleanu, D., Arumugam, V.: On neutral impulsive stochastic differential equations with Poisson jumps. Adv. Differ. Equ. 2018, 290 (2018)

3. Applebaum, D.: Lévy Processes and Stochastic Calculus. Cambridge University Press, Cambridge (2009)

4. Bezandry, P.H., Diagana, T.: Square-mean almost periodic solutions to some stochastic differential equations. Appl. Anal. 86(7), 819-827 (2007)

5. Bezandry, P.H., Diagana, T.: Almost Periodic Stochastic Processes. Springer, New York (2011)

6. Chang, Y., Ma, R., Zhao, Z:: Almost periodic solutions to a stochastic differential equation in Hilbert space. Results Math. 63, 435-449 (2013)

7. Cui, J., Yan, L.: Successive approximation of neutral stochastic evolution equations with infinite delay and Poisson jumps. Appl. Math. Comput. 218, 6776-6784 (2011)

8. Da Prato, G., Tudor, C.: Periodic and almost periodic solutions for semilinear stochastic equations. Stoch. Anal. Appl. $13,13-33(1995)$

9. Hino, Y., Murakami, S., Naito, T.: Functional Differential Equations with Infinite Delay. Lect. Notes Math. (1991)

10. Li, Z., Liu, K., Luo, J.: On almost periodic mild solutions for neutral stochastic evolution equations with infinite delay. Nonlinear Anal. 110, 182-190 (2014)

11. Liu, Z., Sun, K.: Almost automorphic solutions for stochastic differential equations driven by Lévy noise. J. Funct. Anal. 226, 1115-1149 (2014)

12. Mao, W., Hu, L., Mao, X.: Neutral stochastic functional differential equations with Lévy jumps under the local Lipschitz condition. Adv. Differ. Equ. 2017, 57 (2017)

13. Muthukumar, P., Thiagu, K.: Existence of solutions and approximate controllability of fractional nonlocal neutral impulsive stochastic differential equations of order $1<q<2$ with infinite delay and Poisson jumps. J. Dyn. Contro Syst. 23, 213-235 (2017)

14. Pazy, A.: Semigroup of Linear Operators and Applications to Partial Differential Equations. Springer, New York (1992)

15. Ren, Y., Chen, L.: A note on the neutral stochastic functional differential equations with infinite delay and Poisson jumps in an abstract space. J. Math. Phys. 50, 082704 (2009)

16. Ren, Y., Sakthivel, R.: Existence, uniqueness, and stability of mild solutions for second-order neutral stochastic evolution equations with infinite delay and Poisson jumps. J. Math. Phys. 53, 073517 (2012)

17. Sun, K., Wang, Y.: Almost automorphic solutions for stochastic differential equations driven by Lévy noise with exponential dichotomy. Nonlinear Anal. 80(1), 66-75 (2013)

18. Tudor, C.: Almost periodic solutions of affine stochastic evolution equations. Stoch. Stoch. Rep. 38, 251-266 (1992) 
19. Wang, Y., Liu, Z:: Almost periodic solutions for stochastic differential equation with Lévy noise. Nonlinearity 25 , 2803-2821 (2012)

20. Yoshizawa, T.: Stability Theory and Existence of Periodic Solutions and Almost Periodic Solutions. Appl. Math. Sci., vol. 14. Springer, New York (1975)

Submit your manuscript to a SpringerOpen ${ }^{\circ}$ journal and benefit from:

- Convenient online submission

- Rigorous peer review

- Open access: articles freely available online

- High visibility within the field

- Retaining the copyright to your article

Submit your next manuscript at $\gg$ springeropen.com 